\title{
VACINA INATIVADA CONTRA GRIPE TRIVALENTE. ESTUDO COMPARATIVO DA RESPOSTA IMUNITÁRIA PELOS MÉTODOS DE INIBIÇÃO DE HEMAGLUTINAÇÃO E DA HEMOLISE RADIAL SIMPLES
}

\author{
Dalva A. Portari Mancini* \\ Elvira M. Mendes do Nascimento* \\ Valéria Rosa Tavares* \\ Maria Auxiliadora Lucchiari* \\ José Amaral Prado* \\ Murillo A. Soares*
}

\begin{abstract}
MANCINI, D.A.P. et al. Vacina inativada contra gripe trivalente. Estudo comparativo da resposta imunitária pelos métodos de inibição de hemaglutinação e da hemólise radial simples. Rev. Saúde públ., S. Paulo, $19: 438-43,1985$.
\end{abstract}

RESUMO: A vacina inativada contra gripe, trivalente, preparada no Instituto Butantan, contendo 200 unidades hemaglutinantes de cada uma das cepas de virus Influenza A/SP/1/80 (H3N2), A/SP/1/78 (H1N1) e B/England/847/73, foi administrada em 110 voluntários humanos adultos, sendo que 62 receberam uma dose de vacina e 48 duas doses, com intervalo de 21 dias. A resposta de anticorpos específicos para influenza foi analisada comparativamente pelos testes de Inibição da Hemaglutinação (IH) e Hemólise Radial Simples (HRS). Ocorreu aumento significativo do teor de anticorpos nos indivíduos vacinados, correspondente a um aumento de 4 vezes ou mais nos títulos obtidos pelo teste $\mathrm{IH}$ e a um aumento de $3,0 \mathrm{~mm}$ ou maior no diâmetro das zonas de hemólise pelo teste HRS. Os métodos demonstraram correlação satisfatória entre si.

UNITERMOS: Gripe, vacina. Imunização.

\section{INTRODUÇÃO}

Tendo em vista a extrema variabilidade antigênica do vírus influenza ${ }^{6,7,13,15}$, há necessidade de cuidados especiais no controle desta infecção. A vacina inativada contra a gripe vem sendo utilizada na prevenção da influenza. A eficácia imunizante dessa vacina varia de 40 a $80 \%$ (Stuart-Harris e col., $1981^{21}$ ) devido a vários fatores, tais como número de doses inoculadas e antígenos presentes na vacina, ponto crítico na profilaxia contra influenza $1,7,19$, uma vez que esses antígenos devem corresponder aos dos vírus prevalentes na população.

Vários estudos têm sido realizados com o objetivo da avaliação da imunogenicidade da vacina inativada contra a gripe. Em 1976, Smith ${ }^{18}$ encontrou a persistência da imunidade após três anos de vacinação, utilizando a vacina monovalente tipo A. Salk e Salk ${ }^{16}$ (1977) observaram que indivíduos vacinados durante uma epidemia de influenza tipo $\mathrm{A}$ apresentaram imunidade a partir do oitavo e nono dias após a vacinação, e a persistência dessa imunidade foi observada ainda um ano após. A incidência da infecção em grupos parcialmente vacinados foi $85 \%$ menor do que nos grupos não vacinados.

Moffat e col. ${ }^{11}$ (1982) comprovaram a eficácia de uma vacina trivalente contendo as cepas A/Bangkok/1/79 (H3N2), A/Brazil/11/78 (H1N1) e B/Singapore/222/79, em um grupo de 105 voluntários humanos. Ionitã e col. ${ }^{5}$

* Do Serviço de Virologia do Instituto Butantan - Av. Vital Brasil, 1.500 - 05503 - São Paulo, SP Brasil. 
MANCINI, D.A.P. et al. Vacina inativada contra gripe trivalente. Estudo comparativo da resposta imunitária pelos métodos de inibição de hemaglutinação e da hemólise radial simples. Rev. Saúde públ., S. Paulo, 19:438-43, 1985.

(1982), com amostras de soros pareados, antes e após a vacinação, demonstraram aumento significativo de anticorpos para o vírus influenza, pelos testes IH e HRS. Mancini e col $^{8}$ (1982), verificando a resposta de anticorpos para a vacina inativada, em 75 recrutas do exército italiano, na faixa média de 20 anos de idade, observaram aumento de anticorpos em $77 \%$ dos indivíduos estudados, os quais apresentaram títulos de anticorpos inibidores da hemaglutinação $\geqslant$ 1:40 e diâmetro das zonas de hemólise $\geqslant$ a $5,5 \mathrm{~mm}$. Schild e col. ${ }^{17}$ (1975) e Pettersson ${ }^{14}$ (1980), com amostras de soros de indivíduos vacinados, compararam os níveis de anticorpos pelos. métodos de Inibição da Hemaglutinação (IH) e Hemólise Radial Simples (HRS). Verificaram que o teste de HRS oferece maior rapidez e simplicidade.

O presente trabalho visa analisar, através dos testes de IH e HRS, os títulos de anticorpos em soros de 110 indivíduos adultos, voluntários, antes e após uma e duas doses de vacina contra gripe produzida no Instituto Butantan.

\section{MATERIAL E MÉTODOS}

\section{Vacina contra gripe}

A vacina trivalente contra gripe I.B., produzida no Instituto Butantan (São Paulo-Brasil), a partir de ovos embrionados inoculados com vírus influenza, é concentrada pela técnica de Stanley ${ }^{2 n}$ modificada e inativada pelo formol.

A composição da vacina segue as recomendações da Organização Mundial da Saúde ${ }^{13}$, isto é, contém dois tipos $\mathrm{A}$ com genomas diferentes: $A / S P / 1 / 80$ semelhante à $\mathrm{A} / \mathrm{Texas} / 1 / 77$ e $\mathrm{A} / \mathrm{Bang}-$ kok/1/79 (H3N2); A/SP/1/78 semelhante à $\mathrm{A} /$ Brasil/11/78 (H1N1) e um tipo B, B/England $/ 847 / 73$ semelhante à $\mathrm{B} /$ Hong Kong/8/73. O conteúdo antigênico das diversas partidas varia entre 600 a 800 unidades hemaglutinantes, de- terminadas pelo teste da Hemaglutinação. A dose de vacina contém, pelo menos, 200 unidades hemaglutinantes de cada tipo de vírus.

\section{Soros humanos}

Amostras de soro de 110 indivíduos adultos, sem experiência prévia com a vacina contra gripe, com a idade entre 19 a 69 anos, foram obtidos através da coleta do sangue por punção venosa, antes da primeira dose da vacina e 3 semanas após a primeira e/ou segunda doses. Os soros foram inativados a $56^{\circ} \mathrm{C}$ por $30 \mathrm{~min}$. e mantidos congelados a $-20^{\circ} \mathrm{C}$ até a realização dos testes.

\section{Teste da Inibição da \\ Hemaglutinação (IH)}

O teste IH foi realizado de acordo com o método de Takatsy ${ }^{22}$. Inicialmente as amostras de soro foram tratadas com caolim a $20 \%$ em solução tampão fosfato (PBS) e em seguida procedeu-se a titulação.

\section{Teste da Hemólise Radial Simples (HRS)}

$O$ teste de HRS empregado segue a técnica preconizada por Schild e col.17, com modificações ${ }^{3,4,11,12,23}$. Compreendeu o preparo de imunoplacas com uma suspensão a $10 \%$ de hemácias de galo em solução de DGV (Dextrose, Gelatina, Veronal) e volume igual da vacina (antígenos) contendo 200 unidades hemaglutinantes para cada uma das duas cepas do tipo A e 100 unidades hemaglutinantes para o tipo $\mathbf{B}$, constituídos da vacina em estudo.

$\mathrm{Na}$ placa controle, o concentrado viral foi substituído por líquido alantóide puro. Adicionou-se, em seguida, o complemento (soro fresco de cobaia) com 125 unidades - $\mathrm{CH}_{50}$, previamente absorvido com hemácias de galo, e agarose a $1 \%$ fundida e resfriada a $43^{\circ} \mathrm{C}$. Após $30 \mathrm{~min}$. de incubação à tempera- 
MANCINI, D.A.P. et al. Vacina inativada contra gripe trivalente. Estudo comparativo da resposta imunitária pelos métodos de inibição de hemaglutinação e da hemólise radial simples. Rev. Saúde públ., S. Paulo, 19:438-43, 1985.

tura ambiente, foram feitas as perfurações e adicionados os soros inativados. Após $18 \mathrm{~h}$ de incubação a $37^{\circ} \mathrm{C}$ em câmara úmida, foi feita a leitura. Os títulos de anticorpos foram baseados nos diâmetros da zona de hemólise.

\section{RESULTADOS}

Inicialmente comparamos os níveis de anticorpos contra os referidos antígenos de influenza, pelos testes IH e HRS nas 110 amostras de soros antes da vacina- ção, 62 amostras após a primeira dose e 48 amostras após a segunda dose. Os resultados estão demonstrados na Tabela. Verificamos que há uma correspondência geral entre as reações de $\mathrm{IH} e$ HRS.

A distribuição dos diâmetros do halo de hemólise do teste de HRS e dos títulos de anticorpos pelo teste IH podem ser vistos na Figura, antes e após a primeira e segunda doses da vacina contra a gripe.

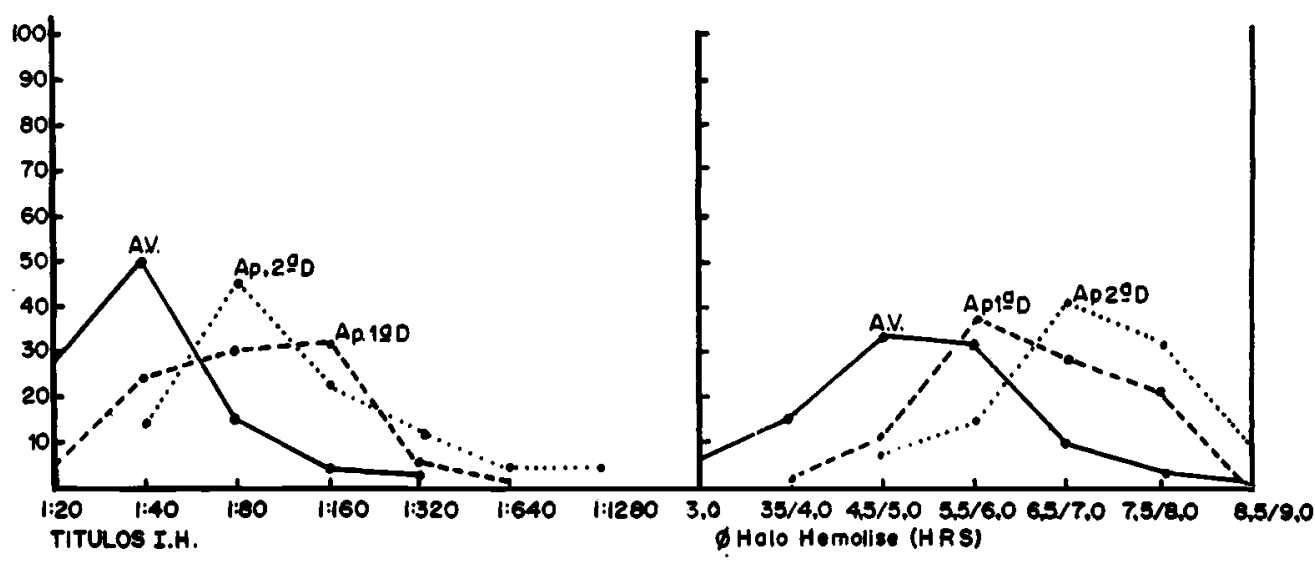

AV-Antes do vocinoczo (llOamostros)

Ap 19O. Após o 19 dose (62 amostras)

Ap $2^{\circ}$ D. Após o $2^{\circ}$ dose (480mostros) 
MANCINI, D.A.P. et al, Vacina inativada contra gripe trivalente. Estudo comparativo da resposta imunitária pelos métodos de inibição de hemaglutinação e da hemólise radial simples. Rev. Saúde públ., S. Paulo, 19:438-43, 1985.

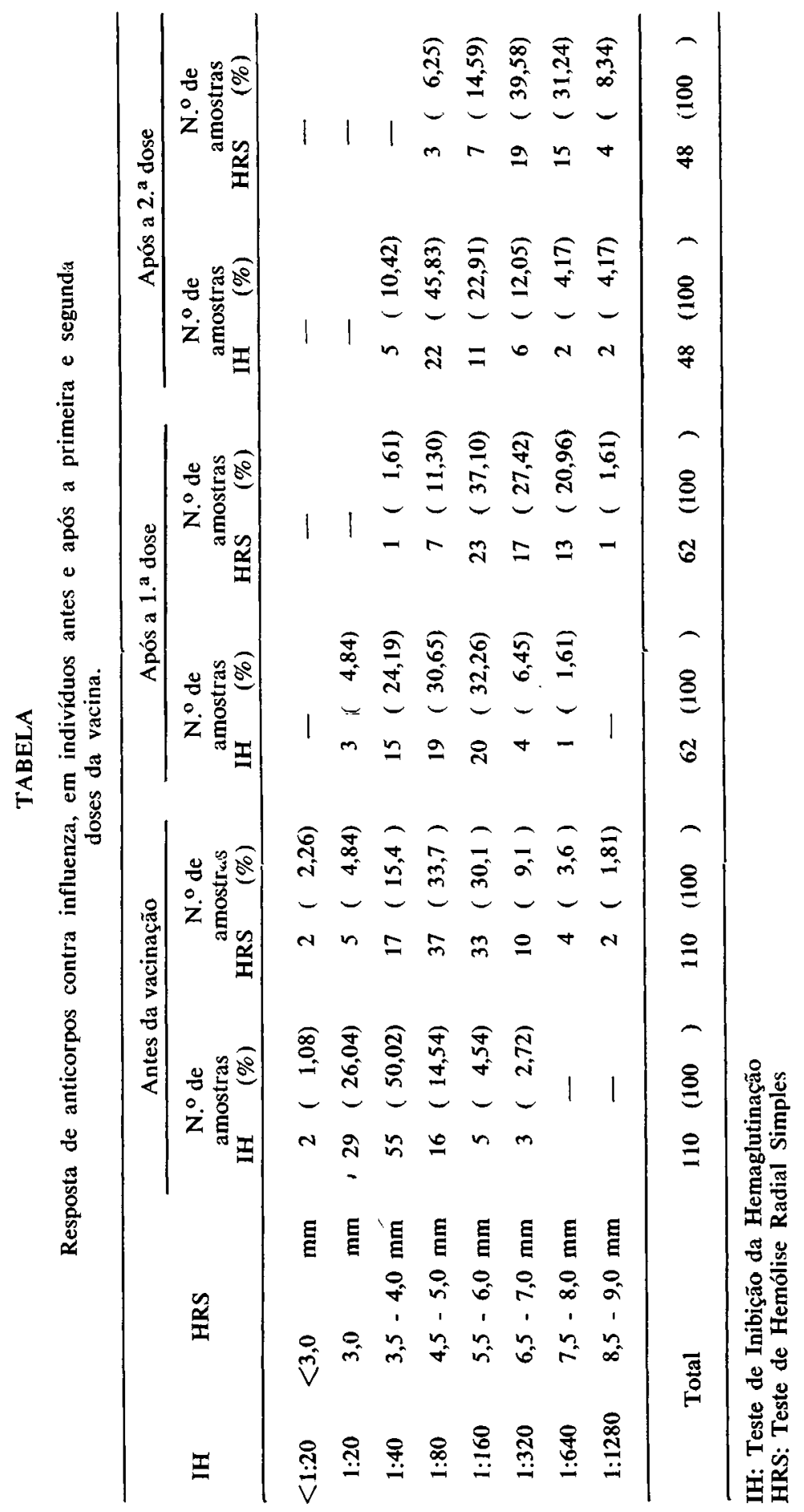


MANCINI, D.A.P. et al. Vacina inativada contra gripe trivalente. Estudo comparativo da resposta imunitária pelos métodos de inibição de hemaglutinação e da hemólise radial simples. Rev. Saúde públ., S. Paulo, 19:438-43, 1985.

\section{DISCUSSÃO E CONCLUSŌES}

Como pode ser visto na Tabela, a vacina em estudo induziu resposta de anticorpos contra os referidos antígenos de influenza, observando-se aumento dos títulos IH e HRS após a primeira e segunda doses. Pode-se observar, também, maior percentagem de títulos da ordem de $1: 1.280$ e $9,0 \mathrm{~mm}$ para IH e HRS, respectivamente, após a segunda dose, ocorrendo aumento de anticorpos, comparados aos iniciais.

$\mathrm{Na}$ Tabela pode-se verificar, ainda, que da vacinação $78,19 \%$ dos soros apresentam títulos de IH iguais ou menores do que $1: 40$, e $21,07 \%$ apresentaram valores iguais ou menores do que $4,0 \mathrm{~mm}$ para HRS. Após a primeira dose, esses valores passam para $29,03 \%$ e $1,61 \%$ de IH e HRS, respectivamente. Após a segunda dose uma diminuição ainda mais acentuada pode ser observada, com apenas $10,42 \%$ dos soros apresentando IH 1:40 e 0\% HRS 4,0 mm.
Observa-se na Figura o deslocamento da distribuição dos títulos IH e dos diâmetros de halos de hemólise HRS.

Os resultados obtidos sugerem haver correlação satisfatória entre os dois métodos e maior sensibilidade do teste HRS, tendo em vista o aparecimento de halos de hemólise na ausência de anticorpos IH.

Podemos afirmar que a vacina produzida no Instituto Butantan promoveu aumento nos títulos de anticorpos nos indivíduos vacinados, confirmando observações de outros autores $5,7,9,10,11$.

Carrere $^{2}$ verificou a persistência de anticorpos, utilizando a vacina contra a gripe IB. Porém, isso não constituiu o objetivo deste trabalho, mas será objeto de estudo posterior.

As recomendações encontradas na literatura internacional e os resultados que temos obtido mostram a importância da imunização contra a gripe, principalmente nos meses que antecedem o inverno, estação de maior incidência das infecções pelos vírus da influenza.

MANCINI, D.A.P, et al. [Inactivated vaccine against trivalent influenza. A comparative study of antigenicity by haemagglutination inhibition and simple radial haemolysis]. Rev. Saúde públ., S. Paulo, $19: 438-43,1985$.

ABSTRACT: The inactivated vaccine anti-influenza virus, prepared at the Butantan Institute, containing $200 \mathrm{U} . \mathrm{H}$. from each one these virus strains, Influenza A/SP/1/80 $\left(\mathrm{H}_{3} \mathrm{~N}_{2}\right), \mathrm{A} / \mathrm{SP} / 1 / 78\left(\mathrm{H}_{1} \mathrm{~N}_{1}\right), \mathrm{B} /$ England/847/73, was administered to 110 human volunteers in one and two doses to 62 and 48 persons respectively, with an interval of 21 days between these doses. The response to specific antibodies to influenza was analysed comparatively by the methods of Haemagglutination-Inhibition (HI) and Single-Radial-Haemolysis (SRH). These methods demonstrated a correlation between themselves, with a percentual correspondence in the negative $H I$ titres, $\leqslant 1: 20$ and diameter of haemolysis $<3.0 \mathrm{~mm}$. Correlation between the haemolysis zone of $3.5 .5 .0 \mathrm{~mm}$ diameter with $\mathrm{HI}$ titres of 1:40-1:80 was observed and titres of $1: 160-1: 320$ by $\mathrm{HI}$ test were equal to haemolysis zones of $5.5 \mathrm{~mm}$ in diameter by SRH test. Very high HI titres $\geqslant 1: 640$, corresponded to hemolysis zones $\geqslant 7.5 \mathrm{~mm}$ in diameter. Soroconversion raie occurred those vaccinated, which corresponded to an increase of 4 times or more in the antibody titres obtained from the HI test and an increase of $3.0 \mathrm{~mm}$ or more in the haemolysis zone diameter.

UNITERMS: Influenza vaccine. Immunization. 
MANCINI, D.A.P. et al. Vlacina inativada contra gripe trivalente. Estudo comparativo da resposta imunitária pelos métodos de inibição de hemaglutinação e da hemólise radial simples. Rev. Saúde públ., S. Paulo, 19:438-43, 1985.

\section{REFERENCIAS BIBLIOGRĀFICAS}

1. ANGELOVA, L.A. Long term immunity to Influenza (H1N1) in humans. Ann. Virol., 133:267-72, 1982.

2. CARRERE, C.M. Medidas preventivas adotadas na TUSA (Transformadores União Ltda.) para controle da gripe: resultados iniciais. [Apresentado na II Jornada de Medicina Ocupacional da Região de Campinas, 1982]

3. CHAKRAVERITY, $P$, et al. Use of the single-radial-diffusion technique for Influenza antibody surveys. Bull. Wld Hlth Org., 49:327-32, 1973.

4. CHAKRAVERITY, P. Comparison of haemagglutination-inhibition and singleradial-haemolysis techniques for detection of antibodies to Influenza B virus. Arch. Virol., 63:285-9, 1980.

5. IONITÃ, E. et al. Use of the hemagglu. tination inhibition with ether treated antigen and of the single-radial-haemolysis methods to detect antibody to Influenza B viruses, Arch, roum. exp. Microbiol., 41:325-30, 1982.

6. KIM, H.W. et al. Influenza $A$ and $B$ virus infection in infants and young children during the years 1957-1976. Amer. J. Epidem., 109:464-79, 1979.

7. MÃGUREANU, E. et al, Epidemiological investigations in young children's communities the efficiency of prophylactic immunization with inactivated Influenza vaccine (I.C.80) in a preepidemic season. Arch. roum. exp. Microbiol. 41:233-8, 1982.

8. MANCINI, G. et al. Resposta anticorpale alla vaccinazione anti-Influenzale trivalente (A Virus Inattivato) A/Texas/1/77 H3N2), A/USSR/90/77 (H1N1) e B/ Hong Kong $/ 8 / 73$, Boll. Ist. Sieroter., Milan, 61(2):85-91, 1982.

9. MACLAREN, C. et al. Comparative antigenicity and immunogenicity of a $\mathrm{A}$ / USSR/77 Influenza vaccines in normal and primed mice. Infect. Immun., 28: 171-7, 1980.

10. MILES, R. et al. A comparative study of the reactogenicity and immunogenicity of two inactivated vaccines in children. J. biol. Stand., 10:59-68, 1982.

11. MOFFAT, M.A.J. et al. Study of the immunogenicity of trivalent inactivated whole-virion Influenza vaccine in hu- man volunteers. J. biol. Stand., 10:83-90, 1982.

12. NASCIMENTO, J.P. et al. Single-radialhaemolysis: a sensitive test for the detection of antibody to Influenza B virus. Rev. Microbiol., 13(1):65-9, 1982.

13. ORGANIZACION MUNDIAL DE LA SALUD. Comite de Expertos en Patrones Biológicos, Ginebra, 1978. 30. $0^{\circ}$ informe. Ginebra, 1979. (Ser. Inf. teen., 638).

14. PETTERSON, R.F, et al. Evaluation of amantadine in the prophylaxis of Influenza A (H1N1) virus infection: a controlled field trial among young adults and high-risk patients. $J$. infect. Dis., 142:377-83, 1980.

15. PONS, V.G. et al. Influenza A/USSR/77 (H1N1) on a university campus. Amer. J. Epidem., 111:23-30, 1980.

16. SALK, J. \& SALK, D. Control of Influen$\mathrm{za}$ and poliomyelitis with killed virus vaccines. Science, 195:834-47, 1977.

17. SCHILD, G.C. et al. Single-radial-haemolysis: a new method for the assay of antibody to Influenza haemagglutinin. Bull. Wld Hlth Org., 52:43-50, 1975.

18. SMITH, J.W.G. Vaccination strategy. In: Selby, P., ed. Influenza: viruses, vaccines, and strategy; proceedings. London, Academic Press, 1976. p. 27 1-94.

19. SMITH, J.W.G. \& POLLARD, R. Vaccination against Influenza: a five-year study in the post-office. J. Hyg., 33:157. 70, 1979.

20. STANLEY, W.M. An evaluation of methods for the concentration and purification of Influenza virus. J. exp. Med., 79:255-66, 1944

21. STUART-HARRIS, C. The epidemiology and prevention of Influenza. Amer. Scient., 69:166-72, 1981.

22. TAKATSY, G. The use of spiral loops in serological and virological micro-methods. Acta microbiol. hung., 3:191, 1955.

23. VAANAREM, $P$. et al. Determination of mumps and Influenza antibodies by haemolysis in gel. Arch. Virol., 52:91-9, 1976.

Recebido para publicaçäo em 20/03/1985 Aprovado para publicação em 08/07/1985 